\title{
Characterization of EPO H131S as a Key Mutation Site in the Hypoxia-adaptive Evolution of Gymnocypris Dobula
}

Congcong Wang ( $\sim$ ccwang@shou.edu.cn )

Shanghai Ocean University

Qin Zhang

Shanghai Ocean University

Yang Liu

Shanghai Ocean University

Qianghua Xu

Shanghai Ocean University

\section{Research Article}

Keywords: EPO, H131S, Gymnocypris dobula, Anti-oxidation, Anti-apoptosis

Posted Date: August 26th, 2021

DOl: https://doi.org/10.21203/rs.3.rs-839445/v1

License: (a) (i) This work is licensed under a Creative Commons Attribution 4.0 International License. Read Full License 


\section{Abstract}

Erythropoietin $(E P O)$ is a glycoprotein hormone involved in proerythropoiesis, antioxidation and antiapoptosis. It also contributes to cellular immune function in high-altitude species, such as the schizothoracine fish Gymnocypris dobula (G. dobula). Six mutation sites previously identified in EPO from $G$. dobula (GD-EPO) were injected into zebrafish embryos and their effects were compared with EPO from the low-altitude schizothoracine Schizothorax prenanti (S. prenanti). The key mutation site in $G D$ EPO was identified as H131S. Under hypoxic conditions, the levels of superoxide dismutase and malondialdehyde were decreased, whereas that of nitric oxide was increased in zebrafish injected with $G D-E P O$ compared with those injected with $S$. prenanti-EPO (SP-EPO). The results suggest that EPO in high-altitude schizothoracine species is both antioxidative and antiapoptotic, driven by the H131S mutation site. Thus, this enhanced the ability of this species to adapt to the high-altitude hypoxic environment. These results provide a basis for investigating further the hypoxia adaptation mechanisms of teleosts.

\section{Introduction}

As the highest plateau in the world, the average altitude of the Tibet Plateau is about $4300 \mathrm{~m}$ with a lower oxygen partial pressure (40\%) compared with that at sea level (Beall 2007; Frisancho 2013). Local species exhibit physiological and morphological characteristics that have evolved to cope with this relatively extreme hypoxic environment. $G$. dobula is a highly specialized schizothoracine fish that mainly inhabits shallow lakes and tributaries at an altitude $>4500 \mathrm{~m}$ (Xu et al. 2016). A recent study reported numerous physiological adaptations in $G$. dobula compared with another schizothoracine, S. prenanti, a relatively primitive fish distributed at a lower altitude in the upper reaches of the Yangtze River (Luo et al. 2016). These adaptations include relatively higher numbers of red blood cells and a stronger hypoxic respiratory response (Beall 2012). However, the detailed regulatory mechanism of these changes was unclear.

Oxidative stress is caused by a variety of environmental factors, including ultraviolet stress, pathogen invasion, and hypoxia (Blokhina 2003). To resist external changes, the organism has developed antioxidant and apoptosis systems (Radi et al. 2014; He et al. 2017). The antioxidant system is mainly composed of small molecular antioxidants and antioxidant enzymes. The main antioxidant enzymes are superoxide dismutase (SOD), malondialdehyde (MDA), catalase (CAT) and so on. (He et al. 2017; Yang et al. 1999). SOD is usually more sensitive than other antioxidant enzymes (Víctor et al. 2009). Oxygen free radicals produced during oxidative stress enable unsaturated fatty acids to form lipid peroxides (MDA). The MDA level does not only reflect the rate and intensity of lipid peroxidation; it also indirectly reflects the degree of tissue peroxidation injury (Tsikas 2017). At physiological levels, nitric oxide (NO) can reduce ROS-induced damage (Wink et al. 2001). As a reactive radical, it is cytotoxic at high concentrations,in mammalian cellular immunity, damage to mitochondria exacerbates cellular lipid peroxidation damage, but at low concentrations, $\mathrm{NO}$ can positively and negatively regulate innate and acquire immune responses (Choudhari et al. 2013). 
There are two main pathways of apoptosis, namely the mitochondrial pathway (endogenous activation pathway) and the death receptor pathway (exogenous activation pathway) (Morrill and He 2017). These pathways share a set of enzymes termed cysteine-aspartic proteases (Caspases), which degrade cellular proteins. Initiator Caspases include Caspase-8 and Caspase-9 (Riedl and Salvesen 2007; Timmer and Salvesen 2007; Obeng 2021). Following an initial apoptotic signal, these enzymes target scaffold proteins. The main executioner Caspases are Caspase-3, Caspase-6, and Caspase-7 (Morrill and He 2014; Obeng 2021). Upon cleavage of executioner Caspases, a proteolytic Cascade begins that will lead to the lysis of nearly all parts of the cell. Caspase- 3 is one of the most important Caspases (Morrill and $\mathrm{He}$ 2017). Moreover, the Bcl-2 family of proteins strongly regulate the intrinsic pathway (Antonsson et al. 1997). Bcl-2 is an integral membrane protein (Chen-Levy et al. 1989; Tsujimoto et al. 1987), but it can also serve as an inhibitor of Cytochrome $\mathrm{c}$ (Cytc) release from the mitochondria as it binds to Bax and inhibits its oligomerization (Yang et al. 1997).

$E P O$ is an acidic glycoprotein hormone of 166 amino acids (Cai 1992). It stimulates the hematopoietic function of bone marrow, and increases red blood cell numbers to improve the oxygen-carrying capacity of blood (Davis et al. 1987; Browne 1986). Meanwhile, Blixt et al. demonstrated that apoptosis, oxidative stress injury, and inflammation in neurocytes were also repressed by EPO, improving the ability to repair hypoxia-induced brain injuries (Katakura et al. 2013). Previous research confirmed the antiapoptotic and antioxidative effects of EPO on tissues or organs in mammals in response to hypoxia and ischemia (Blixt et al. 2018; Tramontano et al. 2003; Parvin et al. 2014; Wang et al. 2010; Chau et al. 2011). Moreover, most mammals exhibit increased levels of plasma EPO promote erythropoiesis, thus improving the physiological response to hypoxia (Souvenir et al. 2011).

The study of hypoxic adaptation in high-altitude fish is limited. G. dobula, one of the most important fish in Tibet, is a good biomaterial to study the hypoxic adaption mechanism of high-altitude fish. We previously reported (Xu et al. 2016) six mutation sites ( $L 1171, H 131 S, T 133 P, S 138 P, S 139 T$, and L153) in $G D-E P O$ compared with $S P$-EPO. We also demonstrated that $G D-E P O$ is involved in improving cell viability in this species. However, it was unclear which mutation site was most important in the adaptation of $G$. dobula to high-altitude hypoxic conditions.

In the current study, we determined the effects of the six mutation sites on zebrafish embryos. Our results provide additional insight into the regulatory mechanisms involved in the adaptation of $G$. dobula to their hypoxic environment.

\section{Materials And Methods}

\section{Animal sources}

Specimens of G. dobula were collected from Yadong County, Tibet $\left(46^{\circ} 03.371^{\prime} \mathrm{N}, 89^{\circ} 17.831^{\prime}\right.$ E, altitude $4506 \mathrm{~m}$; dissolved oxygen $1.9 \pm 0.3 \mathrm{mg} / \mathrm{L} ; 11^{\circ} \mathrm{C}$ ), and $S$. prenanti were collected from Ya'an, Sichuan (altitude $950 \mathrm{~m}$; dissolved oxygen $9.0 \pm 0.5 \mathrm{mg} / \mathrm{L} ; 18^{\circ} \mathrm{C}$ ). Wild-type (WT) zebrafish (AB type), procured 
from Haisheng Biology, Ltd (Shanghai, China), were used as the model organism and were fed freely at $28^{\circ} \mathrm{C}$. The animal experiments were approved by the Ethics Committee of Shanghai Ocean University, and were performed in accordance with the institutional guide for the care and use of laboratory animals.

\section{Q-PCR and construction of recombinant plasmids}

The cDNAs of GD-EPO and SP-EPO were cloned with mRNAs extracted from the pronephros of $G$. dobula (GenBank accession NO: KT188754.1) and S. prenanti (GenBank accession NO. KT188758.1), respectively. In addition, total RNA from zebrafish tissues was extracted using a Total RNA Extraction Kit (Promega, Madison, WI, USA), followed by synthesizing to cDNA using a First-Strand cDNA Synthesis Kit; the cDNA was amplified with a SYBR Green FAST Mastermix (Qiagen, Dusseldorf, Germany). The sequences of primers used are presented in Table 1. GD-EPO and SP-EPO were initially cloned into the empty vector Tol2-bactin -2A-EGFP (Fig. 2b; restriction sites ECOR-I and BamH-I). Meanwhile, six positiveselection amino acid mutation sites ( $L 1171$, H131S, T133P, S138P, S139T, and L153I) from GD-EPO were replaced with those from $S P-E P O$. The $Q 5^{\circledR}$ Site-Directed Mutagenesis Kit was used for PCR in accordance with the manufacturer's instructions. The constructed plasmids (pTOL2-bactin-2A-EGFP, GDEPO, SP-EPO, L117I, H131S, T133P, S138P, S139T, and L1531) which were insert 6xHis Tag. Endotoxin-free plasmids were extracted to determine the concentration and diluted to $50 \mathrm{ng} / \mu \mathrm{L}$. These plasmids were then injected into fertilized zebrafish embryos. After 24-48 h, green fluorescent zebrafish were observed and screened under a fluorescence microscope.

\section{Table 1}

Real-time primers for detecting apoptosis-related gene 


\begin{tabular}{|c|c|c|c|}
\hline Genes & & Primer sequence (5'-3') & Gene Bank \\
\hline \multirow[t]{2}{*}{ ZF- $\beta$-actin } & $\mathrm{F}$ & CACTGAGGCTCCСCTGAATC & \multirow[t]{2}{*}{ NM.131031.2 } \\
\hline & $\mathrm{R}$ & GGGTCACACCATCACCAGAG & \\
\hline \multirow[t]{2}{*}{ ZF-Bcl-2 } & $\mathrm{F}$ & TCTACCGGGTGTTACGGGAT & \multirow[t]{2}{*}{ NM.001030253.2 } \\
\hline & $\mathrm{R}$ & CGTTGCGCAGAATTTGGGTT & \\
\hline \multirow[t]{2}{*}{ ZF-Bax } & $\mathrm{F}$ & CTGGCAGACAGTCGGAGTTT & \multirow[t]{2}{*}{ NM.131562.2 } \\
\hline & $\mathrm{R}$ & GAGGCGGTTTCACCTCTCAA & \\
\hline \multirow[t]{2}{*}{ ZF-Caspase-3 } & $\mathrm{F}$ & CCTGACATCCCAGATGGTCG & \multirow[t]{2}{*}{ NM.131877.3 } \\
\hline & $\mathrm{R}$ & GAGCTCCAGTTCACTGCCAT & \\
\hline \multirow[t]{2}{*}{ ZF-Caspase-8 } & $\mathrm{F}$ & ACCAGGAACAAGGAGGCAGAC & \multirow[t]{2}{*}{ NM_131510.2 } \\
\hline & $\mathrm{R}$ & AATTGTGCCAGCCGAAGAGTT & \\
\hline \multirow[t]{2}{*}{ ZF-Caspase-9 } & $\mathrm{F}$ & AAGATGGATGCCAGTCCGTG & \multirow[t]{2}{*}{ NM_001007404.2 } \\
\hline & $\mathrm{R}$ & TTGTCGCAGTCGATGTTGGA & \\
\hline \multirow[t]{2}{*}{ ZF-Cytochrome C } & $\mathrm{F}$ & GGAGATCGAGGAAACGGCAA & \multirow[t]{2}{*}{ NM_001002068.1 } \\
\hline & $\mathrm{R}$ & TGGCCACGTGTTCAGTTACA & \\
\hline
\end{tabular}

\section{Hypoxia stress}

The screened zebrafish embryos were transferred into a hypoxia water tank (oxygen concentration 10\%). Subsequently, 20 healthy zebrafish (WT) were labeled and added to the same tank. The behavior and mortality of all zebrafish in the tank were observed and recorded at $2,4,8,12,16,24,36$, and $48 \mathrm{~h}$.

\section{O-dianisidine staining}

The constructed plasmids $(50 \mathrm{ng} / \mu \mathrm{L})$ were injected into fertilized zebrafish embryos at the first or second cell stage. After incubation for $48 \mathrm{~h}$, the hemoglobin in the embryos was fixed with $4 \%$ paraformaldehyde for 4-6 h. Subsequently, the residual paraformaldehyde and impurities on the surface of embryos were removed by washing with phosphate buffer ( $1 \times \mathrm{PBS})$ and stained with o-dianisidine [o-dianisidine (0.6 $\mathrm{mg} / \mathrm{mL})$, sodium acetate $(0.01 \mathrm{M}, \mathrm{pH} 4.5), 0.65 \%$ hydrogen peroxide solution, and $40 \%$ ethanol] in the dark for 15-20 min. The dye solution was then discarded and glycerin was added. The stained embryos were observed with glycerol under a light microscope.

\section{Enzyme activity detection}


The levels of superoxide dismutase (SOD), malondialdehyde (MDA), and nitric oxide (NO) were determined in tissue homogenate of WT zebrafish embryos and zebrafish. Zebrafish tissue was homogenized in physiological saline at a mass:volume ratio of 1: 9 ratio to obtain the $10 \%$ homogenate. SOD, MDA, and NO levels were determined respectively using the specific kits (Jiancheng Science \& Technology, Ltd., Nanjing, China), according to the manufacturer's protocol.

\section{Western blotting}

The embryos underwent hypoxia treatment for 6.5 hours at 48 hours post-birth. After washing the embryos with phosphate buffer saline (PBS) three times, the protein productions were extracted using RIPA buffer containing protease inhibitors Proteins were then separated by $12 \%$ SDS-PAGE gel and transferred onto a PVDF membrane. Blocking was performed using $5 \%$ nonfat dried milk. After blocking, membranes were incubated with primary antibodies against Bcl-2 (DIA. An, China), Bax (DIA. An, China), Caspase-3 (DIA. An, China), 6x His (HUABIO, China), and GAPDH (DIA. An, China) for $2.5 \mathrm{~h}$ at room temperature. Then, $1 \times$ PBST was used to wash the membranes three times. Subsequently, at room temperature, a secondary antibody (DIA. An, China) was added and incubated for $1 \mathrm{~h}$. The membranes were then washed three times with $1 \times$ PBST. The membranes were developed by Chemiluminescence reagents (Thermo Fisher Scientific, USA) under a Gel-Pro analyzer (version 4.0, USA). Finally, the western blotting images were analyzed using the ImageJ 1.46 software (National institutes of Health, USA).

\section{Statistical analysis}

All results were obtained from at least three independent experiments. Statistical analyses were performed using GraphPad Prism 8.0.2. Data were expressed as the mean \pm standard deviation. One-way ANOVA was used to evaluate the differences among multiple groups. The survival rate of zebrafish was obtained by Percent Survival analysis. Differences were significant at $P<0.05$ or $P<0.01$.

\section{Results And Discussion}

\section{GD-EPO increases the expression level of hemoglobin under hypoxia}

$E P O$ is a glycoprotein hormone with a principal regulatory role in erythropoiesis (Spivak 1989; Fried 2009). Various studies on red blood cells and hemoglobin in species from the Tibet plateau and plain areas showed that the oxygen transport and utilization abilities of these species are stronger than those living at lower altitude areas, manifesting as large lung volume, high myoglobin concentration, and strong oxygen uptake ability (Yangzong et al. 2013; Li et al. 2018). In the current study, injection of nine overexpressed plasmids in zebrafish embryos increased the hemoglobin level in the acute hypoxic group compared with the normoxia group, suggesting that hypoxia increases the numbers of red blood cells, 
leading to increased levels of hemoglobin (Fig. 1). However, the nine overexpressed plasmids in zebrafish embryos of the normoxia group had no obvious effect on hemoglobin level (Fig. 1b). By contrast, in the hypoxia groups, the expression level of hemoglobin in zebrafish embryos injected with GD-EPO was significantly elevated compared with those injected with SP-EPO (Fig. 1a). This suggests that, under hypoxia stress, GD-EPO may increases the level of hemoglobin to carry more oxygen and overcome the effects of hypoxia. In addition, the hemoglobin level was upregulated by overexpression of $L 117$, T133P, S138P, S139T, and L153/ compared with control group and overexpression of SP-EPO, whereas there were no obvious changes in the overexpressed H131S group (Fig. 1a). Thus, the mutant site H131S might have a crucial role in enabling $G$. dobula to adapt to a high-altitude hypoxia environment.

\section{H131S is a key mutation site in EPO in the hypoxia-adaptive evolution of $G$. dobula}

Six amino acid mutation sites ( $L 117$ I, H131S, T133P, S138P, S139T, H131S, and L153I) were previously identified in GD-EPO compared with SP-EPO using a human EPO model (Fig. 2a). In the current study, $\mathrm{H} 131 \mathrm{~S}$ was determined to have a key role in the protective mechanism of GD-EPO against a hypoxic environment. To further validate this hypothesis, the percentage survival of zebrafish injected with $G D$ EPO, SP-EPO, L117I, H131S, T133P, S138P, S139T, H131S, and L153/ was assessed. The $4 \mathrm{~h}$ percentage survival of zebrafish in the GD-EPO, $L 1171, T 133 P, S 138 P, S 139 T$, and $L 153 /$ groups was $100 \%, 100 \%, 80 \%$, $60 \%, 70 \%$, and $30 \%$, respectively (Fig. 2c). By contrast, numerous zebrafish in the H131S group had died 2 $\mathrm{h}$ after hypoxia stimulation, with $0 \%$ survival at $4 \mathrm{~h}$. This suggests that $\mathrm{H} 131 \mathrm{~S}$ overexpression in zebrafish renders them more sensitive to oxygen concentration. Previously, we reported that the H131S of GD-EPO lacks a CK2 phosphorylation site (serine/threonine protein kinase), which regulates the activity or stability of the substrate via its phosphorylation (Xu et al. 2016). CK2 also participates in the development and function of neurons, and in synaptic information transmission, which controls the development and longevity of synaptic connections. Therefore, the CK2 phosphorylation site might be missing at the 131 amino acid site of $G$. dobula, rendering it insensitive to hypoxia. Thus, mutated $H 131 S$ might have enabled $G$. dobula to adapt to its high-altitude hypoxic environment.

\section{GD-EPO functions as an antioxidant}

The Tibet Plateau is characterized by low temperatures, hypoxia, and strong radiation (Beall 2007). Dramatic hypoxia has significant negative effects on organisms, including metabolic disorders, increasing concentrations of oxygen-derived free radicals, damage to cell membranes and nucleic acid structures, or even death (Fuhrmann and Brune. 2017; Gonzalez et al. 2019) . To adapt to hypoxic environments, organisms have evolved endogenous antioxidant enzyme systems and apoptosis, comprising small-molecule antioxidants and antioxidant enzymes. SOD is a cytoplasmic and mitochondrial-based antioxidant that can convert $\mathrm{O}_{2}{ }^{-}$into $\mathrm{H}_{2} \mathrm{O}_{2}$ and $\mathrm{O}_{2}$ (Du et al. 2017). Oxygen-derived free radicals produced during oxidative stress can convert unsaturated fatty acids to MDA (Tsikas 2017). 
Generally, the MDA level indirectly reflect the degree of tissue peroxidation injury (Yang et al. 1999). In addition, $\mathrm{NO}$ as a reactive free radical functions in nerve transmission, the immune response, and apoptosis (Ghasemi 2019). Humans living on the Tibetan Plateau have a relatively high concentration of NO, which suggest that NO has an important role in this hypoxic environment (Beall 2007). In this study, the levels of SOD, NO, and MDA in injected zebrafish embryos under hypoxia conditions were determined. Levels of SOD and MDA in zebrafish embryos injected with GD-EPO were significantly decreased compared with those of WT zebrafish embryos $(P<0.05)$ or those injected with $S P$-EPO $(P<0.05)$ (Fig. $3 \mathrm{~b}$ and d). Similarly, Omrani et al. reported that the levels of SOD and MDA in rat tissues were increased significantly after hypoxia stimulation (Omrani et al. 2017). Manor et al. also confirmed that the levels of ROS and lipid oxidation in mice injected with EPO (5000 U/kg) were decreased (Manor et al. 1986). These results suggest that $G D-E P O$ not only alleviated the oxidative damage caused by hypoxia, thus reducing the production of free radicals, but also attenuated the degree of lipid oxidation. In addition, a high level of NO occurred in zebrafish embryos injected with GD-EPO compared with WT zebrafish embryos or those injected with SP-EPO (Fig. 3c). In Tibetans, the utilization of NO is relatively high compared with plain inhabitants. Thus, the antioxidant effect of EPO might be closely associated with the hypoxic environment of the Tibet Plateau.

\section{GD-EPO functions as a role of antiapoptosis}

Under conditions of ischemia and hypoxia, the mitochondrial membrane potential is decreased and mitochondrial permeability transition pores (MPTP) open, inducing the expression of apoptosis-related genes and proteins (Chen et al. 2018). Cytc is a water-soluble protein located between the inner and outer mitochondrial membranes (Wan et al. 2019). Mitochondria release Cytc into the cytoplasmic matrix to initiate apoptosis once cells are stimulated by apoptosis signals (Wan et al. 2019). Members of the Bcl-2 protein family can interact to induce or prevent apoptosis. Among them, Bcl-2 inhibits the opening of mPTPs, thereby repressing apoptosis. By contrast, Bax promotes the opening of mPTP to accelerate apoptosis (Zhang et al. 2015). In this study, to investigate the role of GD-EPO in apoptosis, the expression levels of Cytc, Caspase-3, Caspase-8, Caspase-9, and the Bcl-2/Bax ratio were determined. Expression levels of Cytc, Caspase-3, Caspase-8, and Caspase- 9 in the GD-EPO groups were reduced compared with those in the $S P$-EPO group $(P<0.01)$ (Fig. $4 \mathrm{a}$ and b), whereas the Bcl-2/Bax ratio was higher in the $G D$ $E P O$ group, as also shown by western blotting (Fig. 4c). EPO interacts with the p53 signaling pathway ( Pham et al. 2019) in leukemia cells or the Akt signaling pathway (Tramontano et al. 2003) in cardiac myocytes to suppress apoptosis. Antiapoptotic treatments, such as EPO, are also suggested to improve outcomes in hypoxic brain injury (Jung et al. 2021). EPO and vitamin D3 (VD3) can be used to prevent or treat renal ischemia-reperfusion (I/R) injury, and these beneficial effects are closely related to antiinflammatory and anti-apoptosis pathways (Qin et al. 2021). Thus, we hypothesize that EPO is also involved in the regulation of apoptosis in $G$. dobula through these pathways. However, the specific mechanisms involved remain to be fully understood. 


\section{Conclusions}

In summary, the H131S mutation was identified as a key mutation affecting the cytoprotective function of G. dobula EPO during the process of adaptation to long-term hypoxia. Our results showed that EPO from high-altitude fish could increase the hemoglobin level. Furthermore, the antioxidative and antiapoptotic functions of G. dobula EPO were enhanced during evolution. Although the detailed regulatory mechanism remains unclear, these results provide a research direction to elucidate the mechanisms utilized by schizothoracine fish under long-term hypoxia conditions.

\section{Declarations}

\section{Acknowledgements}

We would like to thank all of the people in the Yadong Forestry Bureau, Tibet, for the sample collection.

\section{Author contribution}

Qin Zhang carried out most of the experiments. Congcong Wang designed the methods and experiments, interpreted the results and finished the discussion. Yang Liu prepared the materials and partly worked on the experiments. Qianghua Xu was responsible for overall supervision, and participated in coordination. All authors have read and approved the final manuscript.

\section{Funding}

This work is supported by the National Natural Science Foundation of China (Grant no. 31702312), the National Key R\&D Program of China (Grant No. 2018YFD0900601) and the National Natural Science Foundation of China (Grant no. 31772826).

\section{Data availability}

The data used to support the findings of this study are available from the corresponding author upon request.

\section{Ethics approval}

Experimental protocols involving live animals were approved by the Ethics Committee for the Use of Animal Subjects of Shanghai Ocean University.

\section{Consent for publication}


All authors consent to participate in this publication.

\section{Conflict of interest}

The authors declare that they have no conflict of interest.

\section{References}

1. Antonsson B, Conti F, Ciavatta A, Montessuit S, Lewis S, Martinou I, Bernasconi L, Bernard A, Mermod JJ, Mazzei G, Maundrell K, Gambale F, Sadoul R, Martinou JC (1997) Inhibition of Bax channelforming activity by Bcl-2. Science 277(5324): 370-372. https://doi.org/10.1126/science.277.5324.370

2. Beall CM (2007) Two routes to functional adaptation: Tibetan and Andean high-altitude natives. Proc Natl Acad Sci 104 (S1): 8655-8660. https://doi.org/10.1073/pnas.0701985104

3. Beall CM, Laskowski D, Erzurum SC (2012) Nitric oxide in adaptation to altitude. Free Radic Biol. Med 52(7):1123-34. https://doi.org/ 10.1016/j.freeradbiomed.2011.12.028

4. Blixt J, Gunnarson E, Wanecek M (2018) Erythropoietin Attenuates the Brain Edema Response after Experimental Traumatic Brain Injury. J Neurotrauma 35(4): 671-680. https://doi.org/ 10.1089/neu.2017.5015

5. Blokhina O, Virolainen E, Fagerstedt KV (2003) Antioxidants, Oxidative Damage and Oxygen Deprivation Stress: a Review. Annals of Botany 91(2): 179-194. https://doi.org/10.1093/aob/mcf118

6. Browne JK, Cohen AM, Egrie JC, Lai PH, Lin FK, Strickland T, Watson E, Stebbing N (1986) Erythropoietin: gene cloning, protein structure, and biological properties. Cold Spring Harb Symp Quant Biol 51(1): 693-702. https://doi.org/10.1101/SQB.1986.051.01.082

7. Cai YL Chen HM, Min YJ, Zhang J, Wang QS (1992) Synthesis and cloning of the whole human erythropoietin (EPO) gene. Acta Academiae Medicinae Sinicae 14: 173-178

8. Chau M, Chen D, Wei L (2011) Erythropoietin Attenuates Inflammatory Factors and Cell Death in Neonatal Rats with Intracerebral Hemorrhage. Intracerebral Hemorrhage Research 111: 299-305. https://doi.org/10.1007/978-3-7091-0693-8_50

9. Chen-Levy Z, Nourse J, Cleary ML (1989) The bcl-2 candidate proto-oncogene product is a 24kilodalton integral-membrane protein highly expressed in lymphoid cell lines and lymphomas carrying the $t(14 ; 18)$ translocation. Molecular and cellular biology 9(2): 701-710. https://doi.org/10.1128/mcb.9.2.701

10. Chen GH, Yang YJ, Xu CS, Gao S (2018) A Flow Cytometry-based Assay for Measuring Mitochondrial Membrane Potential in Cardiac Myocytes After Hypoxia/Reoxygenation. J Vis Exp 137: e57725. https://doi.org/10.3791/57725

11. Choudhari SK, Chaudhary M, Bagde S, Gadbail AR, Joshi V (2013) Nitric oxide and cancer: a review. World J Surg Oncol 11: 118. https://doi.org/ 10.1186/1477-7819-11-118 
12. Davis JM, Arakawa T, Strickland TW, Yphantis DA (1987) Characterization of recombinant human erythropoietin produced in Chinese hamster ovary cells. Biochemistry 26: 2633-2638. https://doi.org/10.1021/bi00383a034

13. Du J, Cai J, Wang ST, You H (2017) Oxidative stress and apotosis to zebrafish (Danio rerio) embryos exposed to perfluorooctane sulfonate (PFOS) and ZnO nanoparticles. International Journal of Occupational Medicine and Environmental Health 30(2): 213-229. https://doi.org/10.13075/ijomeh.1896.00669

14. Fried W (2009) Erythropoietin and erythropoiesis. Exp Hematol 37: 1007-1015. https://doi.org/10.1016/j.exphem.2009.05.010

15. Frisancho AR (2013) Developmental Functional Adaptation to High Altitude: Review. Am J Hum Biol 25: 151-168. https://doi.org/10.1002/ajhb.22367

16. Fuhrmann DC, Brune B (2017) Mitochondrial composition and function under the control of hypoxia. Redox Biol 12: 208-215. https://doi.org/10.1016/j.redox.2017.02.012

17. Ghasemi M (2019) Nitric oxide: Antidepressant mechanisms and inflammation. Adv Pharmacol 86: 121-152. https://doi.org/10.1016/bs.apha.2019.04.004

18. Gonzalez FJ, Xie C, Jiang C (2019) The role of hypoxia-inducible factors in metabolic diseases. Nat Rev Endocrinol 15: 21-32. https://doi.org/10.1038/s41574-018-0096-z

19. He L, He T, Farrar S, Ji LB, Liu TY, Ma X (2017) Antioxidants Maintain Cellular Redox Homeostasis by Elimination of Reactive Oxygen Species. Cell Physiol Biochem 44(2): 532-553. https://doi.org/10.1159/000485089

20. Jung S, Terörde K, Dörr HG, Trollmann R (2021) Recombinant Human Growth Hormone Activates Neuroprotective Growth Factors in Hypoxic Brain Injury in Neonatal Mice. Endocrinology 162: 3, bqab008. https://doi.org/10.1210/endocr/bqab008

21. Katakura F, Katzenback BA, Belosevic M (2013) Molecular and functional characterization of erythropoietin of the goldfish (Carassius auratus L.). Developmental \& Comparative Immunology 40: 148-157. https://doi.org/10.1016/j.dci.2013.02.0071

22. Li CY, Li XW, Liu J, Fan X, You GX, Zhao L, Zhou H, Li JQ, Lei HF (2018) Investigation of the differences between the Tibetan and Han populations in the hemoglobin-oxygen affinity of red blood cells and in the adaptation to high-altitude environments. Hematology 23(5): 309-313. https://doi.org/10.1080/10245332.2017.1396046

23. Luo H, Xiao SJ, Ye H, Zhang ZS, Lv CH, Zheng SM, Wang ZY, Wang XQ (2016) Identification of Immune-Related Genes and Development of SSR/SNP Markers from the Spleen Transcriptome of Schizothorax prenanti. PLOS ONE 11(3): e0152572. https://doi.org/10.1371/journal.pone.0152572

24. Manor D, Fibach E, Goldfarb A, Rachmilewitz EA (1986) Erythropoietin activity in the serum of beta thalassemic patients. Scand J Haematol 37: 221-228. https://doi.org/10.1111/j.16000609.1986.tb02301.x

25. Morrill S, He DZZ (2017) Apoptosis in inner ear sensory hair cells. J Otol 12(4): 151164. https://doi.org/10.1016/j.joto.2017.08.001 
26. Obeng E (2021) Apoptosis (programmed cell death) and its signals - A review. Brazilian Journal of Biology 81(4): 1133-1143. https://doi.org/10.1590/1519-6984.228437

27. Omrani H, Alipour MR, Farajdokht F, Ebrahimi H, Abbasi. MM, Mohaddes. G (2017) Effects of Chronic Ghrelin Treatment on Hypoxia-Induced Brain Oxidative Stress and Inflammation in a Rat Normobaric Chronic Hypoxia Model. High Alt. Med. Biol, 18: 145-151. https://doi.org/10.1089/ham.2016.0132

28. Parvin A, Pranap R, Shalini U, Devendran A, Baker JE, Dhanasekaran A (2014) Erythropoietin protects cardiomyocytes from cell death during hypoxia/reperfusion injury through activation of survival signaling pathways. PLoS One 9(9): e107453. https://doi.org/10.1371/journal.pone.0107453

29. Pham TD, Ma W, Miller D, Kazakova L, Benchimol S (2019) Erythropoietin inhibits chemotherapyinduced cell death and promotes a senescence-like state in leukemia cells. Cell Death Dis 10: 22. https://doi.org/10.1038/s41419-018-1274-6

30. Qin LY, Lin X, Liu J, Dong R, Yuan J, Zha Y (2021) The combination of vitamin D3 and erythropoietin alleviates acute kidney injury induced by ischemia-reperfusion via inhibiting inflammation and apoptosis. Basic Medical Sciences 24: 167-174. https://doi.org/10.22038/ijbms.2020.51384.11661

31. Radi E, Formichi P, Battisti C, Federico A (2014) Apoptosis and oxidative stress in neurodegenerative diseases. J Alzheimers Dis 42 (S3): S125-S152. https://doi.org/10.3233/JAD-132738

32. Riedl SJ, Salvesen GS (2007) The apoptosome: signaling platform of cell death. Nat Rev Mol Cell Biol 8: 405-413. https://doi.org/10.1038/nrm2153

33. Souvenir R, Fathali N, Ostrowski RP, Lekic T, Zhang JH, Tang J (2011) Tissue inhibitor of matrix metalloproteinase-1 mediates erythropoietin-induced neuroprotection in hypoxia ischemia. Neurobiol Dis 44: 28-37. https://doi.org/10.1016/j.nbd.2011.05.020

34. Spivak JL (1989) Erythropoietin. Blood Rev 3(2): 130-135. https://doi.org/10.1016/0268960X(89)90008-8

35. Timmer JC, Salvesen GS (2007) Caspase substrates. Cell Death Diff 14: 66-72. https://doi.org/10.1038/sj.cdd.4402059

36. Tramontano AF, Muniyappa R, Black AD, Blendea MC, Cohen I, Deng L, Sowers JR, Cutaia MV, ElSherif N (2003) Erythropoietin protects cardiac myocytes from hypoxia-induced apoptosis through an Akt-dependent pathway. Biochemical \& Biophysical Research Communications 308(4): 990-994. https://doi.org/10.1016/s0006-291x(03)01503-1

37. Tsujimoto Y, Ikegaki N, Croce CM (1987) Characterization of the protein product of bcl-2, the gene involved in human follicular lymphoma. Oncogene 2(1): 3-7. Tsikas D (2017) Assessment of lipid peroxidation by measuring malondialdehyde (MDA) and relatives in biological samples: Analytical and biological challenges. Anal Biochem 524: 13-30. https://doi.org/10.1016/j.ab.2016.10.021

38. Víctor VM, Espulgues JV, Hernández-Mijares A, Rocha M (2009) Oxidative stress and mitochondrial dysfunction in sepsis: a potential therapy with mitochondria-targeted antioxidants. Infect Disord Drug Targets 9(4): 376-89. https://doi.org/10.2174/187152609788922519

39. Wang HJ, Jiang HL, Chen XH, Lin PY, Zhu YC, Tao LL (2010) The anti-apoptosis effect of erythropoietin on neonatal rat cardiocytes during hypoxia/reoxygenation injury and its possible 
mechanism. Chinese Critical Care Medicine, 22(5): 302-5.

40. Wan J, Kalpage HA, Vaishnav A, Liu J, Lee I, Mahapatra G, Turner AA, Zurek MP, Ji Q, Moraes CT, Recanati MA, Grossman LI, Salomon AR, Edwards BFP, Hüttemann M (2019) Regulation of Respiration and Apoptosis by Cytochrome c Threonine 58 Phosphorylation. Sci Rep 9: 15815. https://doi.org/10.1038/s41598-019-52101-z

41. Wink DA, Miranda KM, Espey MG, Pluta RM, Hewett SJ, Colton C, Vitek M, Feelisch M, Grisham MB (2001) Mechanisms of the antioxidant effects of nitric oxide. Antioxid Redox Signal 3(2): 203-13. https://doi.org/: 10.1089/152308601300185179

42. Xu QH, Zhang C, Zhang DS, Jiang HP, Peng SH, Liu Y, Zhao K, Wang CC, Chen LB (2016) Analysis of the erythropoietin of a Tibetan Plateau schizothoracine fish (Gymnocypris dobula) reveals enhanced cytoprotection function in hypoxic environments. BMC Evolutionary Biology 16:11. https://doi.org/10.1186/s12862-015-0581-0

43. Yang J, Liu X, Bhalla K, Kim CN, Ibrado AM, Cai J, Peng TI, Jones DP, Wang X (1997) Prevention of apoptosis by Bcl-2: release of cytochrome c from mitochondria blocked. Science 275 (5303): 11291132. https://doi.org/10.1126/science.275.5303.1129

44. Yang RH, Chen JY, Liu XD, Deng ZY, Liu XH (1999) Lipid peroxide damage in retinal ganglion cells induced by microwave. Journal of Hygiene Research, 28(4): 200-202

45. Yangzong, Berntsen S, Bjertness E, Stigum H, Gonggalanzi, Bianba, Nafstad P (2013) Lung function among 9- to 10-year-old Tibetan and Han Chinese schoolchildren living at different altitudes in Tibet. High Alt Med Biol 14(1): 31-36. https://doi.org/10.1089/ham.2012.1034

46. Zhang FB, Song XW, Li L, Wang JF, Lin LY, Li C, Li HT, Lv YJ, Jin YH, Liu Y, Hu Y, Xin T (2015) Polygala tenuifolia polysaccharide PTP induced apoptosis in ovarian cancer cells via a mitochondrial pathway. Tumour Biol 36: 2913-2919. https://doi.org/10.1007/s13277-014-2921-x

\section{Figures}


a

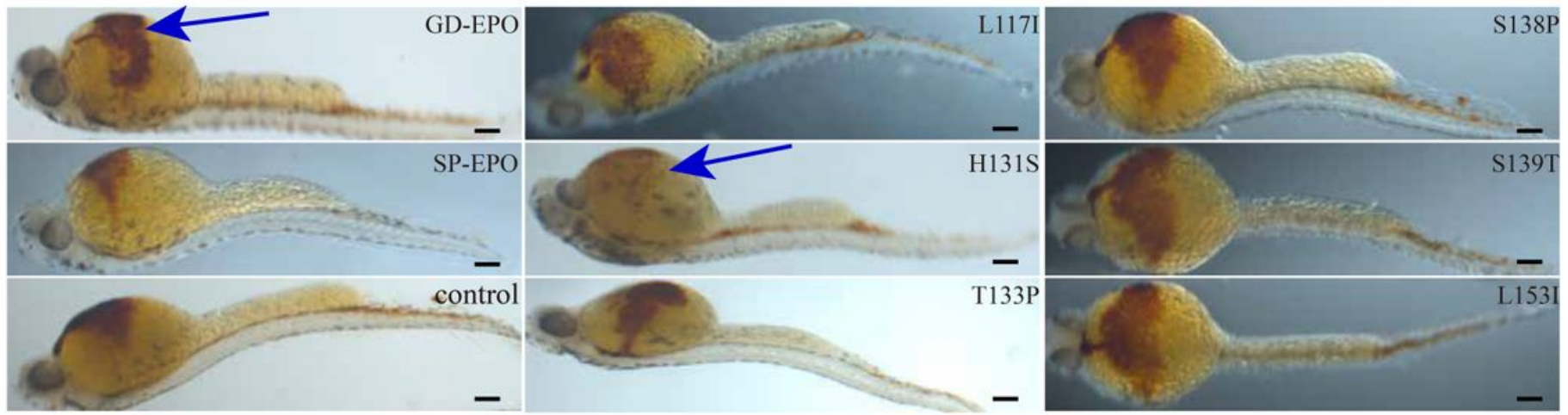

b

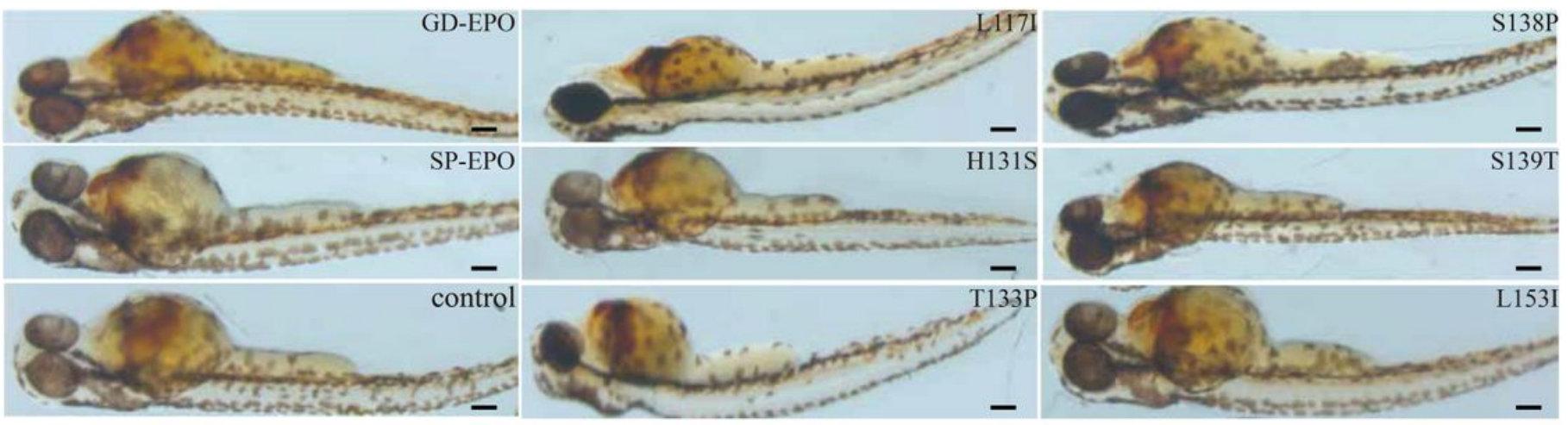

\section{Figure 1}

GD-EPO increases the expression level of hemoglobin under hypoxia. (a) Overexpression of GD-EPO, L117I, T133P, S138P, S139T and L153I significantly enhanced the expression of hemoglobin , and in contrast to overexpression of SP-EPO and H131S based on O-dianisidine staining at 10\% oxygen, the blue arrow indicate hemoglobin expression. (b) Overexpression of eight EPO genes were similar with control on expression of hemoglobin under normoxia.囚The scale is $40 \mu \mathrm{m}$ ) 
a

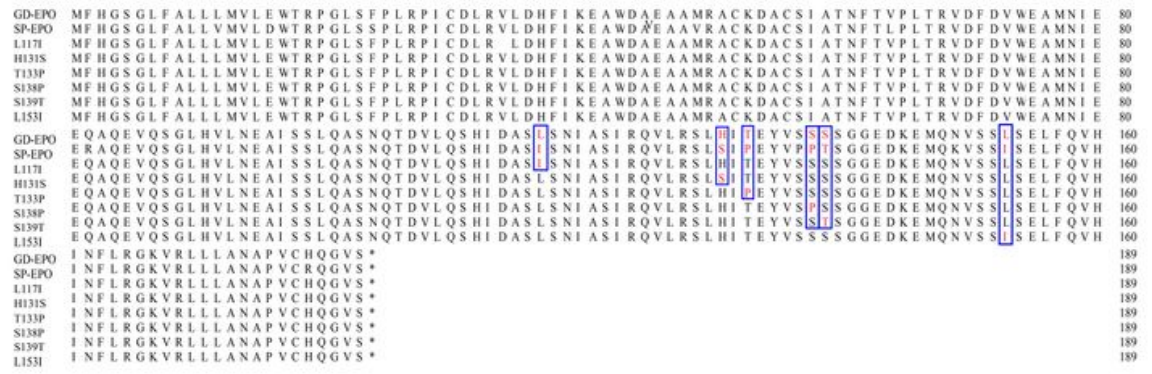

c

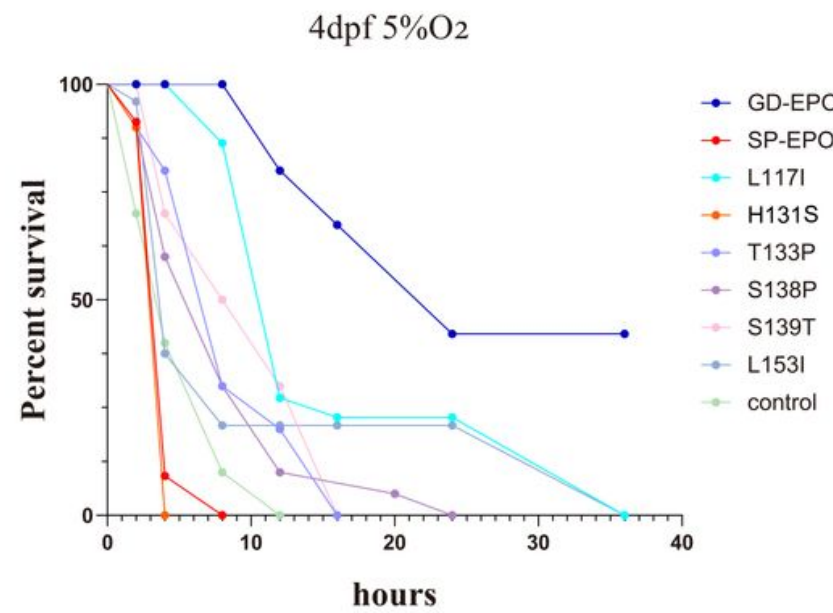

b

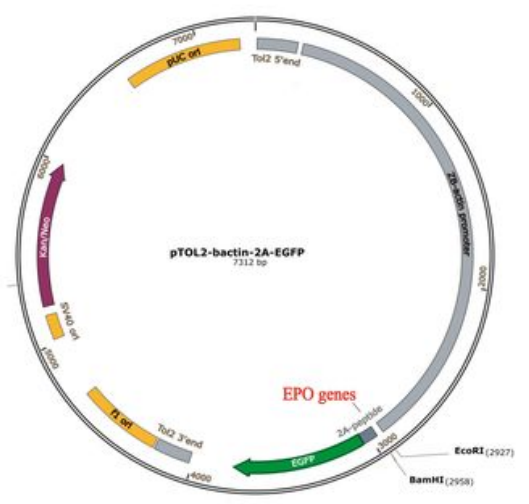

$6 \mathrm{XHis}$

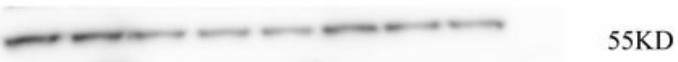

GAPDH

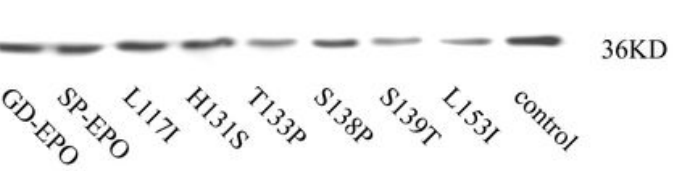

\section{Figure 2}

$\mathrm{H} 131 \mathrm{~S}$ is a key mutation site in EPO in the hypoxia-adaptive evolution of G. dobula. (a) Eight EPO sequences, including GD-EPO, SP-EPO, L117I, H131S, T133P, S138P, S139T, and L153I, six positive selection mutation sites for GD and SP are marked in red lable and highlighted with blue rectangle. (b) The schematic drawing of the recombinant pTOL2-bactin-EPO-2A-EGFP plasmid. The coding sequences of GD-EPO or SP-EPO were subcloned into the PTOL2-bactin-2A-EGFP vector digested with ECOR-I and BamH-I. EPO was co-expressed with pTOL2-bactin-2A-EGFP. (c) Survival rate of zebrafish under hypoxia and the relative viability (\%) was estimated as the percentage. (d) Western blotting analysis fusion of EPO-6xHis-EGFP and GAPDH. 


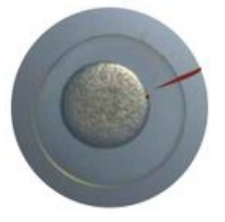

c

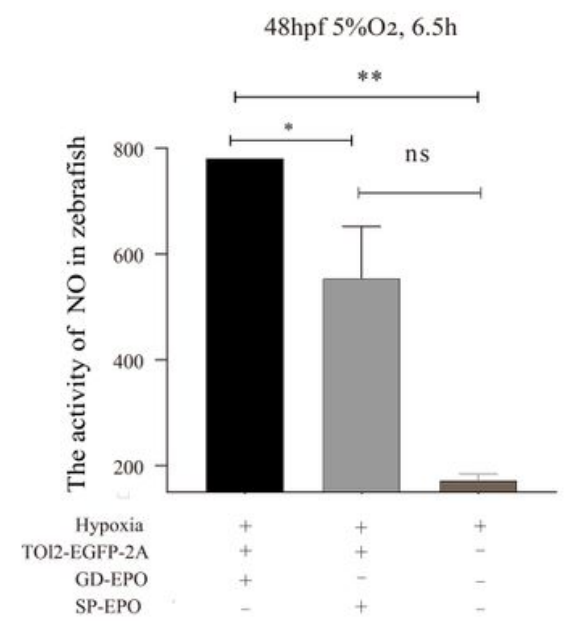

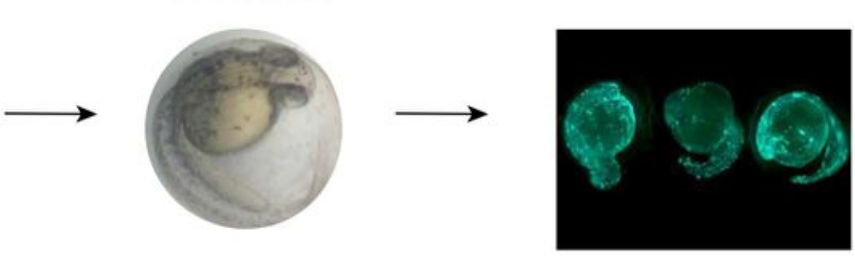

d
48 hpf $5 \% \mathrm{O}_{2}, 6.5 \mathrm{~h}$

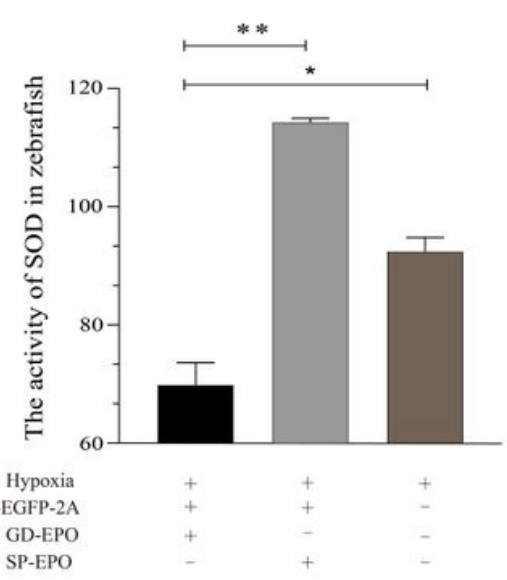

Figure 3

GD-EPO functions as a role of antioxidant. (a) The flow chart for obtaining GD-EPO and SP-EPO zebrafish. The recombinant plasmid (pTOL2-bactin-GD-EPO-2A-EGFP, pTOL2-bactin-SP-EPO-2A-EGFP) were injected into the zebrafish embryo, and the green fluorescent zebrafish was detected and screened after $24 \mathrm{~h}$. (b-d) The SOD, NO, MDA activities in the 4 dpf zebrafish under hypoxia. "+"yes, "-"none, All data were expressed as the mean \pm SD of three independent experiments. ${ }^{*} p<0.05$, ${ }^{\star *} p<0.01$. 
$\mathrm{a}$

$48 \mathrm{hpf} 10 \% \mathrm{O} 2,6.5 \mathrm{~h}$
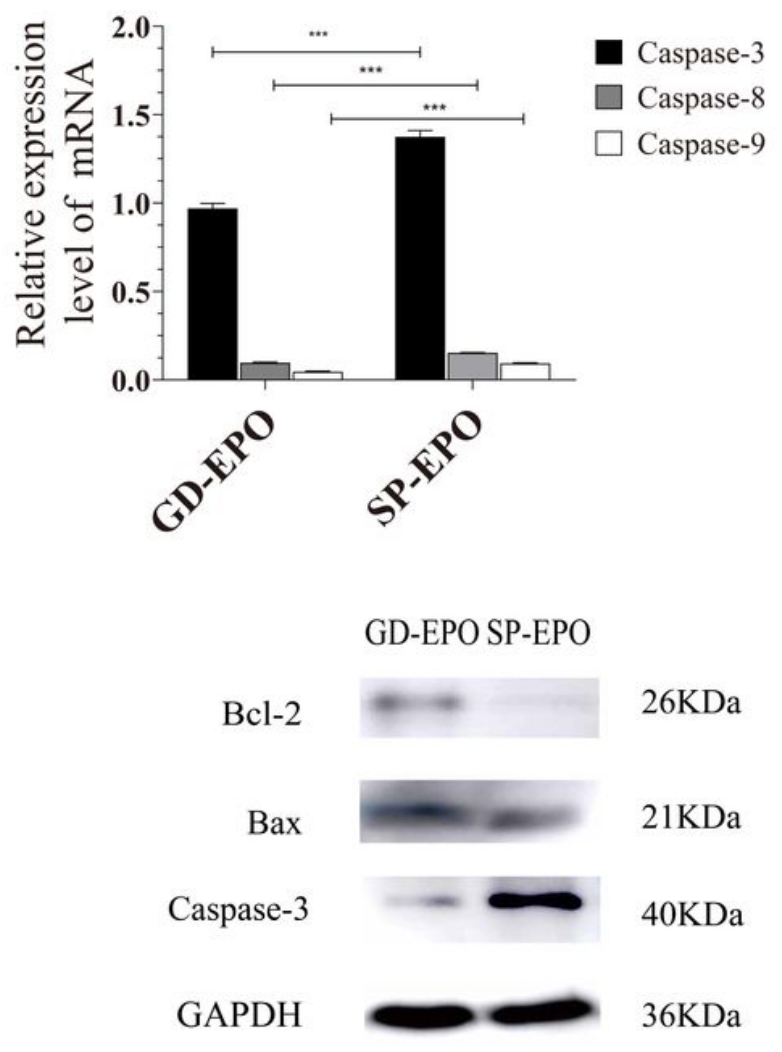

$\mathrm{b}$

$48 \mathrm{hpf} 10 \% \mathrm{O} 2,6.5 \mathrm{~h}$
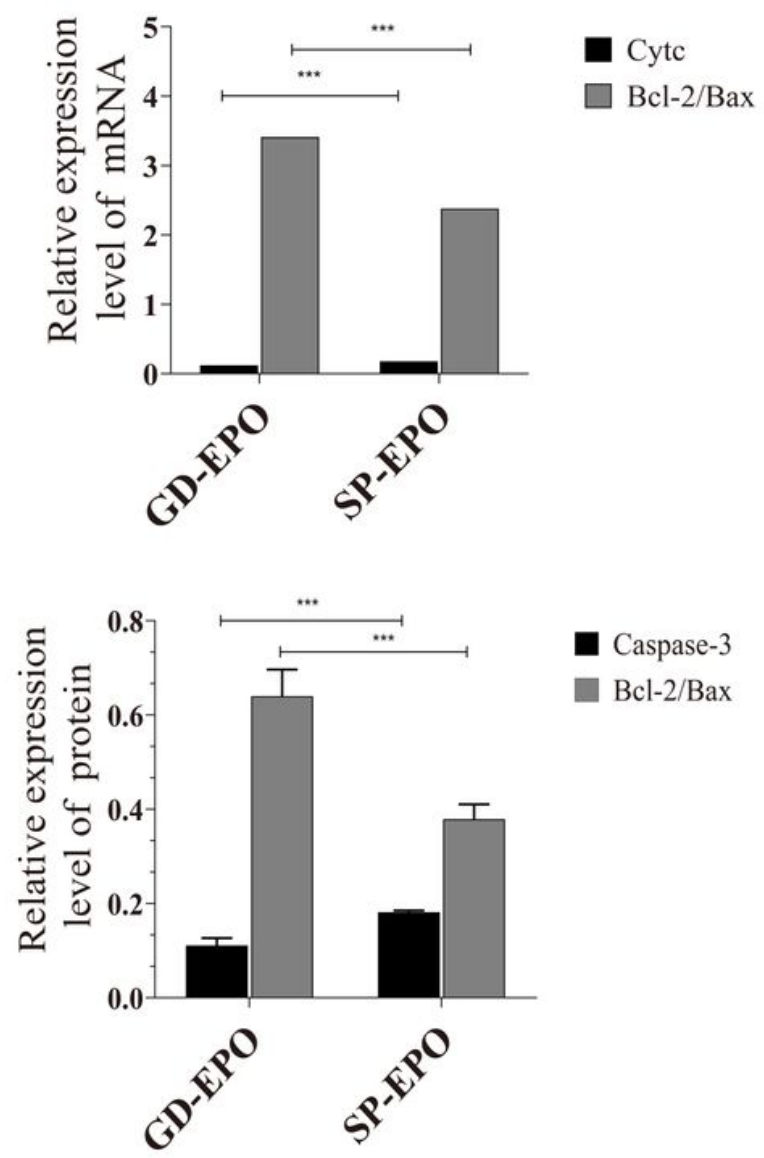

Figure 4

GD-EPO can inhibit the expression of apoptosis-related genes. (a-b) The mRNA level of Caspase-3, Caspase-8, Caspase-9, Cytc, Bax and Bcl-2. (c) Expression level of Bcl-2, Bax, Caspase-3 by Western blotting, The results were expressed as the mean \pm SD of three independent experiments. ${ }^{*} p<0.05$ and $\star * x<0.01$. 